\title{
HdBe STARS: HYDROGEN-DEFICIENT SUPERGIANTS WITH EMISSION LINES
}

\author{
C.S. JEFFERY \\ Dept of Physics and Astronomy, University of St Andrews, North Haugh, St Andrews, \\ FIFE KY16 9SS, Scotland
}

\section{The hydrogen-deficient Be stars}

The hydrogen-deficient B stars (often known as extreme-helium stars: EHes) show weak or absent Balmer lines and the following properties (Heber 1986a): $T_{\text {eff }} \sim 12-25 \mathrm{kK}, \log g \sim 2-3, \log n_{\mathrm{H}} \sim-3--4, n_{\mathrm{He}} \approx 0.99, n_{\mathrm{C}} \sim$ 0.01 . Their galactic positions and apparent magnitudes indicate that they are low-mass supergiants belonging to the Galactic bulge (Jeffery et al. 1987).

All EHes have been observed using high-resolution échelle spectrographs (at ESO and the AAT). A small number show an emission-line spectrum, usually consisting of $\mathrm{He}_{\mathrm{I}}, \mathrm{C}_{\text {II }}$ and $\mathrm{Si}_{\text {II }}$ lines; other lines including Balmer lines are occasionally seen. Whilst the spectra vary from object to object, the general characteristics (see table) and specific behaviour (Jeffery \& Heber 1992) resemble that observed in normal Be stars (Balmer lines excepted). The term 'Hydrogen-deficient Be stars' refers to these objects.

\section{Origin of the emission lines}

From the type of ions detected, the stellar $T_{\text {eff }}$ and the relative strengths in the $\mathrm{He}_{\mathrm{r}}$ lines, the emission lines are deemed to arise in an extended atmosphere (shell) with a temperature similar to or slightly below that of the photosphere.

One object, $\mathrm{BD}-9^{\circ} 4395$, has a low surface gravity close to the Eddington limit, a relatively high rotational velocity $\left(v_{\text {eq }} / v_{\text {crit }}>0.36\right)$, pulsations (Jeffery et al. 1985, Jeffery \& Heber 1992) and a radiatively driven wind (Hamman et al. 1981). Although less well studied, LSE 78 and DY Cen show similar properties. It is reasonable to suppose that the combination of high luminosity, rapid rotation and pulsations supports an extended atmosphere.

\section{Related objects}

Although extremely rare, the EHes may be related to other hydrogendeficient stars, including RCrB stars, late-type Wolf-Rayets (WC10,11), $\mathrm{H}$-deficient subdwarf $\mathrm{O}$ stars (HdsdO) and planetary nebula central stars 
(HdCPN), and PG1159 stars. Some of these (see table) have similar $T_{\text {eff }}$ to the EHes. They differ in having stronger emission spectra with $\mathrm{P}$ Cygni profiles (e.g. V348 Sgr: Leuenhagen \& Hamman 1993). It will be proposed elsewhere (Jeffery, in preparation) that although qualitatively different, HdBes, WC11s, RCrBs, HdsdOs, HdCPNs and PG1159s share a common evolutionary origin, probably as a white dwarf (possibly binary) which experiences helium-shell reignition. Diversification depends critically on the structure of the progenitor(s) and the process leading to helium-shell ignition.

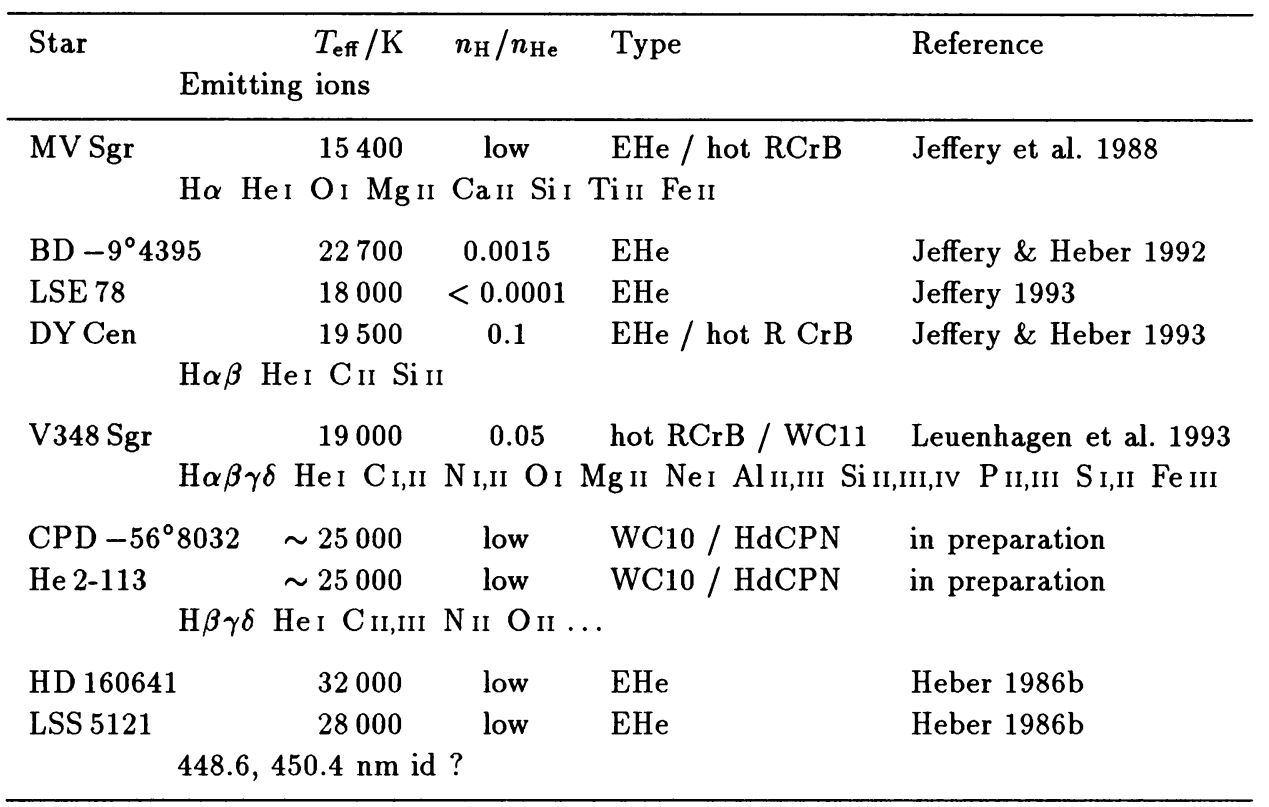

\section{References}

Hamann W.-R., Heber U., Schönberner D.: 1981, $A A$ 116, 273

Heber U.: 1986a, "' in Hunger, Schönberner \& Rao, ed(s)., Hydrogen-deficient Stars and Related Objects, IAU Coll. 87, Reidel:Dordrecht, 33

Heber U.: 1986b, "' in Hunger, Schönberner \& Rao, ed(s)., Hydrogen-deficient Stars and Related Objects, IAU Coll. 87, Reidel:Dordrecht, 73

Jeffery C.S.: 1993, $A A$, in press

Jeffery C.S., Heber,U.: 1992, $A A$ 260, 133

Jeffery C.S., Heber U.: 1993, AA 270, 167

Jeffery C.S., Heber U., Hill P.W., Pollacco D.: 1988, MNRAS 231, 175

Jeffery C.S., Skillen I., Hill P.W., Kilkenny D., Malaney R.A., Morrison K.: 1985, MNRAS 217, 701

Leuenhagen U., Hamann W.-R.: 1993, $A A$, submitted

Leuenhagen U., Heber U., Jeffery C.S.: $1993, A A S$, in press 